\title{
¿Qué trae la nueva clasificación de las neumonías intersticiales idiopáticas?
}

\section{What does the new classification of Idiopathic Interstitial Pneumonias bring?}

Jorge PULIDo ARENAS, MD.

Las neumonías intersticiales idiopáticas son un grupo de enfermedades parenquimatosas pulmonares que comparten múltiples características entre ellas, pero que son 10 suficientemente diferentes entre sí para ser designadas como entidades separadas (1).

Dentro de este grupo se incluyen la neumonía intersticial usual (NIU), la neumonía intersticial inespecífica (NII), la bronquiolitis respiratoriaenfermedad pulmonar intersticial (BR-EPI), la neumonía criptogénica en organización (NOC), la neumonía intersticial aguda (NIA), la neumonía intersticial linfoide (NIL) y la fibroelastosis pleuroparenquimatosa idiopática (FEPP). El diagnóstico se obtiene de la combinación metódica y detallada de la historia clínica, el examen físico, los estudios de laboratorio, las imágenes diagnósticas y el estudio histopatológico, lo que permite diferenciar estas entidades de otras formas de enfermedad parenquimatosa difusa pulmonar (2).

El término idiopático se reserva para aquellas condiciones donde permanece desconocida la causa de la lesión pulmonar, descartando entidades frecuentes como enfermedades del tejido conectivo, reacciones a medicamentos, asbestosis y neumonitis por hipersensibilidad, entre otras $(1,2)$.

Desde la última actualización de 2002 de la ATS/ERS sobre la clasificación de las neumonías intersticiales idiopáticas, hay un progreso sustancial en varios aspectos (tablas 1 у 2) (3).

El abordaje diagnóstico de las neumonías intersticiales idiopáticas es multidisciplinario y dinámico. Requiere de la comunicación cercana entre el clínico, el radiólogo y el patólogo (1). Dentro de los datos clínicos es de vital importancia conocer los síntomas de presentación, los antecedentes exposicionales, la historia de tabaquismo previo y actual, las comorbilidades presentes, la función pulmonar y los hallazgos en pruebas de laboratorio y radiológicas (3). Esta aproximación multidisciplinaria no le resta importancia a la biopsia pulmonar para el diagnóstico de las NII; por el contrario, define los escenarios en los cuales es suficiente con los hallazgos de la tomografía computarizada de alta resolución (TCAR) o si existe indicación de biopsia pulmonar. La concordancia entre observadores con relación al diagnóstico de las
Internista. Departamento de Medicina Interna. Hospital Universitario San Ignacio. Pontificia Universidad Javeriana. Bogotá, Colombia.

Correspondencia: Jorge Pulido Arenas. Correo electrónico: jpulidoarenas@ gmail.com

Recibido: 12-03-2013. Aceptado: 02-06-2013. 
neumonías intersticiales depende de la experiencia del clínico y de la integración de la información por parte de las otras especialidades, resaltándose la importancia de que estos pacientes sean evaluados por equipos multidisciplinarios con experiencia y en centros hospitalarios de referencia $(4,5)$.

Como se mencionó inicialmente, es muy importante y a veces difícil el diagnóstico diferencial de estas entidades, pues suelen confundirse con la neumonitis por hipersensibilidad y viceversa. El abordaje multidisciplinario, como se describió previamente, es fundamental para la diferenciación entre $\mathrm{NH}$ y las neumonías intersticiales idiopáticas, particularmente la FPI y la NII (6). El análisis cuidadoso de los hallazgos en la TCAR sumado a la histopatología, ayudan al diagnóstico, siendo esencial la búsqueda detallada del factor desencadenante, aunque hasta el 30\% de los pacientes no tiene una exposición claramente identificable (7). De igual forma, el diagnóstico diferencial con las enfermedades del colágeno vascular son causa frecuente de patrones de neumonía intersticial, especialmente NII. Sin embargo, los hallazgos clínicos, serológicos, radiológicos e histológicos son útiles para establecerlo.

Otra entidad que amerita mención son las neumonías intersticiales familiares (NIF). Continúan siendo clasificadas como neumonías intersticiales idiopáticas pese a que son secundarias a mutaciones heterocigóticas de múltiples genes (SFTPC, SFTPA2, TERT, TERC) hasta en el 20\% de los casos (8). La presentación entre casos de neumonía intersticial familiar y esporádica es indistinguible en la TCAR y la biopsia. Por tanto, en quienes se sospeche neumonía intersticial idiopática debe indagarse por sus antecedentes familiares relevantes y, de acuerdo a esto, dirigir la búsqueda de mutaciones genéticas puntuales y de otros miembros de la familia potencialmente comprometidos (9).

En cuanto a la última clasificación de las neumonías intersticiales idiopáticas de la ATS/ERS de 2013, es de gran importancia la inclusión de la NII como una entidad clínico-patológica específica. Los criterios diagnósticos de las NII fueron mencionados recientemente por la ATS (10). La NII ocurre como una entidad idiopática pero también en situaciones tan diversas como las enfermedades del colágeno, $\mathrm{NH}$, toxicidad por medicamentos y en algunos pacientes con fibrosis pulmonar familiar. Es en estos escenarios en los que el abordaje multidisciplinario es de vital importancia (10). El pronóstico de la enfermedad es variable, oscilando entre la mejoría y la progresión a fibrosis terminal que conlleva a la muerte del paciente $(11,12)$.

En la FPI existe un nuevo algoritmo diagnóstico (13) que incluye tres niveles de certeza para los hallazgos radiológicos de NIU en la TCAR (NIU, NIU posible, inconsistente con NIU) y cuatro niveles de certeza para los hallazgos histopatológicos (NIU, probable, posible y ausente). Para el diagnóstico se requiere la exclusión de otras causas de enfermedad pulmonar intersticial, la presencia de un patrón de NIU en la TCAR en aquellos pacientes no susceptibles de biopsia pulmonar o la combinación de hallazgos en TCAR e histopatológicos en quienes fueron llevados a biopsia pulmonar. El hallazgo de FPI con hallazgos definitivos de NIU en la TCAR, conlleva una supervivencia menor que aquellos pacientes con TCAR indeterminada $(14,15)$.

Desde la última revisión en 2002 se ha acumulado nueva información importante en el grupo de las NII asociadas al tabaquismo. En general, la BR-EPI es una enfermedad de fumadores con exposición promedio de más de 30 paquetes/año (16). Bien se sabe que es un hallazgo histológico presente en todos los fumadores activos y puede considerarse un cambio fisiológico secundario al estímulo nocivo del humo del tabaco, pero en algunos individuos progresa en extensión para convertirse en una enfermedad intersticial. En la práctica clínica actual, la BR-EPI es una entidad que se diagnostica cada vez con más frecuencia sin necesidad de biopsia pulmonar en aquellos pacientes fumadores con las características radiológicas descritas y con lavado broncoalveolar que muestre macrófagos pigmentados en ausencia de linfocitosis (17). El curso de esta enfermedad es heterogéneo, con una minoría de pacientes que progresa a pesar de suspender el hábito de fumar (18).

Otra entidad conocida en esta categoría es la NID, un tipo de neumonía intersticial poco común, que aparece comúnmente en hombres fumadores, entre 
30 a 40 años de edad aunque ha sido reconocida en no fumadores; al parecer está relacionada como la continuidad de una NID de la infancia en la edad adulta asociada a mutaciones en genes relacionados con el surfactante pulmonar (19).

En el grupo de las neumonías intersticiales idiopáticas agudas o subagudas figuran la NCO y la NIA. La primera continúa incluida en la clasificación por su naturaleza idiopática y su frecuente confusión con otras formas de neumonías intersticiales, especialmente cuando hay progresión a fibrosis (3). La NIA es una condición de naturaleza idiopática, de curso rápido similar al síndrome de dificultad respiratoria aguda (SDRA) con una mortalidad cercana al 50\% y que no tiene tratamiento específico. Los hallazgos radiológicos e histológicos en la fase aguda son indistinguibles del patrón histológico del SDRA (1).

De otra parte, está claramente establecido que en las neumonías intersticiales idiopáticas puede haber exacerbaciones agudas; actualmente se tiene mayor reconocimiento y mejor definición en la FPI y la NII (3). Dentro de estas, la FPI es la que más muestra este comportamiento. Sin embargo, en todas las situaciones es importante descartar causas secundarias de descompensación pulmonar aguda como infección, falla ventricular izquierda, neumotórax y tromboembolia pulmonar antes de atribuir los síntomas a una exacerbación aguda (20).

Así mismo, se ha creado una categoría adicional de neumonías intersticiales idiopáticas raras, dentro la cual se incluyen la NIL y la FEPP y aquellos patrones histológicos inusuales como la neumonía en organización fibrinosa aguda (NOFA) y la neumonía intersticial con patrón bronquiolocéntrico (3).

Pese a los avances y el abordaje multidisciplinario exhaustivo, la categoría de neumonías intersticiales idiopáticas no clasificables aplica para aquellas entidades en las que no se puede llegar a un diagnóstico final o que presentan sobreposición de patrones histológicos. De acuerdo con un estudio de cohorte reciente, este grupo representa el 10\% de los pacientes evaluados en un centro de referencia de enfermedades pulmonares intersticiales (21). En estos casos el tratamiento debe dirigirse al diagnóstico más probable y al comportamiento esperado de la enfermedad.
Por último, la identificación de biomarcadores útiles para el diagnóstico, manejo y pronóstico es materia de investigaciones actuales. Altos niveles séricos de algunas proteínas epiteliales asociadas a macrófagos como la SP-A, SP-D, KL-6, CCL18 y MMP-7 se han asociado con deterioro rápido de la función pulmonar y/o supervivencia disminuida (22-25). Estas asociaciones requieren validación, pero sugieren que pueden existir herramientas adicionales útiles para identificar pacientes con alto riesgo de progresión.

En conclusión, el campo de las neumonías intersticiales idiopáticas cuenta con gran dinámica actual en investigación. Es muy importante la referenciación de los pacientes a centros con experiencia en estas patologías, conformados por equipos médicos multidisciplinarios donde la pericia de clínicos, radiólogos y patólogos es fundamental para efectuar un diagnóstico correcto, así como clasificar y tratar adecuadamente a los pacientes. Finalmente, es deseable desarrollar un algoritmo para la clasificación y manejo de los casos, sobre todo en aquellas situaciones en que no se dispone de histopatología y los hallazgos radiológicos en la TCAR no son diagnósticos.

Se esperan importantes avances en los próximos años respecto al descubrimiento, la implementación y el desarrollo de estudios genéticos y moleculares que revolucionen la aproximación diagnóstica a las neumonías intersticiales idiopáticas y permitan establecer el pronóstico de este grupo de enfermedades.

Tabla 1. Clasificación de las neumonías intersticiales idiopáticas de acuerdo con la ATS/ERS.

\begin{tabular}{|l|}
\hline \multicolumn{1}{|c|}{ Neumonías intersticiales idiopáticas principales } \\
\hline Neumonía intersticial aguda \\
\hline Neumonía criptogénica en organización \\
\hline Neumonía intersticial descamativa \\
\hline Bronquiolitis respiratoria-enfermedad pulmonar \\
\hline intersticial \\
\hline Neumonía intersticial idiopática inespecífica \\
\hline Fibrosis pulmonar idiopática \\
\hline Neumonías intersticiales idiopáticas raras \\
\hline Fibroelastosis pleuroparenquimatosa idiopática \\
\hline Neumonía intersticial linfoide \\
\hline Neumonías intersticiales idiopáticas no clasificables \\
\hline
\end{tabular}


Tabla 2. Categorización de las neumonías intersticiales idiopáticas principales.

\begin{tabular}{|l|l|l|}
\hline \multicolumn{1}{|c|}{ Categoría } & Diagnóstico clínico-radiológico-patológico & \multicolumn{1}{c|}{$\begin{array}{c}\text { Patrones radiológicos y/o histopatológicos } \\
\text { asociados }\end{array}$} \\
\hline \multirow{2}{*}{ Aguda/subaguda } & Neumonía intersticial aguda & Daño alveolar difuso \\
\cline { 2 - 3 } & Neumonía criptogénica en organización & Neumonía en organización \\
\hline \multirow{4}{*}{ Asociadas al tabaquismo } & Neumonía intersticial descamativa & Neumonía intersticial descamativa \\
\cline { 2 - 3 } & $\begin{array}{l}\text { Bronquiolitis respiratoria-enfermedad } \\
\text { pulmonar intersticial }\end{array}$ & Bronquiolitis respiratoria \\
\hline \multirow{3}{*}{ Crónicas fibrosantes } & Fibrosis pulmonar idiopática & Neumonía intersticial usual \\
\cline { 2 - 3 } & $\begin{array}{l}\text { Neumonía intersticial idiopática } \\
\text { inespecífica }\end{array}$ & Neumonía intersticial inespecífica \\
\hline
\end{tabular}

\section{Bibliografía}

1. American Thoracic Society, European Respiratory Society. American Thoracic Society/European Respiratory Society international multidisciplinary consensus classification of the idiopathic interstitial pneumonias. Am J Resp Crit Care Med. 2002;165:277-304.

2. Hobbs S, Lynch D. The idiopathic interstitial pneumonias: an update and review. Radiol Clin N Am. 2014;52:105-20.

3. Travis WD, Costabel U, Hansell DM, King TE Jr, et al. An official American Thoracic Society/European Respiratory Society statement: update of the international multidisciplinary classification of the idiopathic interstitial pneumonias. Am J Resp Crit Care Med. 2013;188:733-48.

4. Flaherty KR, Andrei AC, King TE Jr, et al. Idiopatic interstitial pneumonia: do community and academic physicians agree on diagnosis? Am J Respir Crit Care Med. 2007;175:1054-60.

5. Flaherty KR, King TE Jr, Raghu G, et al. Idiopathic interstitial pneumonia: what is the effect of a multidisciplinary approach to diagnosis? Am J Respir Crit Care Med. 2004;170;904-10.

6. Selman M. Hypersensitivity pneumonitis: a multifaceted deceiving disorder. Clin Chest Med. 2004;25:531-47.

7. Hanak V, Golbin JM, Ryu JH. Causes and presenting features in 85 consecutive patients with hypersensitivity pneumonitis. Mayo Clin Proc. 2007;82:812-16.

8. van Moorsel CH, van Oosterhout MF, Barlo NP, de Jong PA, van der Vis JJ, Ruven HJ, et al. Surfactant protein C mutations are the basis of a significant portion of adult familial pulmonary fibrosis in a Dutch cohort. Am J Respir Crit Care Med. 2010;182:1419-25.

9. Lawson WE, Loyd JE, Degryse AL. Genetics in pulmonary fibrosisfamilial cases provide clues to the pathogenesis of idiopathic pulmonary fibrosis. Am J Med Sci. 2011;134:439-3.

10. Travis WD, Hunninghake G, King TE Jr, Lynch DA, Colby TV, Galvin JR, et al. Idiopathic nonspecific interstitial pneumonia: report of an American Thoracic Society Project. Am J Respir Crit Care Med. 2008;177:1338-47.

11. Akira M, Inoue Y, Arai T, Okuma T, KAwata Y. Long-term follow-up high-resolution CT findings in non-specific interstitial pneumonia. Thorax. 2011;66:61-5.

12. Park IN, Jegal Y, Kim DS, Do KH, Yoo B, Shim TS, et al. Clinical course and lung function change of idiopathic nonspecific interstitial pneumonia. Eur Respir J. 2009;33:68-76.

13. Raghu G, Collard HR, Egan JJ, Martinez FJ, Behr J, Brown KK, Colby TV, Cordier JF, Flaherty KR, Lasky JA, et al. ATS/ERS/JRS/ALAT Committee on Idiopathic Pulmonary Fibrosis. An official ATS/ERS/
JPS/ALAT statement: idiopathic pulmonary fibrosis: evidence-based guidelines for diagnosis and management. Am J Respir Crit Care Med. 2011;183:788-824

14. Sumikawa H, Johkoh T, Colby TV, Ichikado K, Suga M, Taniguchi H, et al. Computed tomography findings in pathological usual interstitial pneumonia: relationship to survival. Am J Respir Crit Care Med. 2008;177:433-9.

15. Lynch DA, Godwin JD, Safrin S, Starko KM, Hormel P, Brown KK, et al. Idiopathic Pulmonary Fibrosis Study Group. High-resolution computed tomography in idiopathic pulmonary fibrosis: diagnosis and prognosis. Am J Respir Crit Care Med. 2005;172:488-93.

16. Fraig M, Shreesha U, Savici D, et al. Respiratory bronchiolitis: a clinicopathologic study in current smokers, ex-smokers, and never-smokers. Am J Surg Pathol. 2002;26:647-53.

17. Vasallo R, Ryu JH. Tobacco smoke-related diffuse lung diseases. Semin Respir Crit Care. 2008;29:643-50.

18. Portnoy J, Veraldi KL, Schwartz MI, Cool CD, Currant-Everett D, Cherniack RM, et al. Respiratory bronchiolitis-interstitial lung disease: longterm outcome. Chest. 2007;131:664-71.

19. Doan ML, Guillerman RP, Dishop MK, Nogee LM, Langston C, Mallory $\mathrm{GB}$, et al. Clinical, radiological and pathological features of ABCA3 mutations in children. Thorax. 2008;63:366-73.

20. Collard HR, Moore BB, Flaherty KR, Brown KK, Kaner RJ, King TE $\mathrm{Jr}$, , et al. Idiopathic Pulmonary Fibrosis Clinical Research Network Investigators. Acute exacerbations of idiopathic pulmonary fibrosis. Am J Respir Crit Care. Med 2007;176:636-43.

21. Ryerson CJ, Urbania TH, Richeldi L, et al. Prevalence and prognosis of unclassifiable interstitial lung disease. Eur Respir J. 2013;42:750-7.

22. Barlo NP, van Moorsel CHM, Ruven HJT, Zanen P, van der Bosch JMM, Grutters JC. Surfactant protein-D predicts survival in patients with idiopathic pulmonary fibrosis. Sarcoidosis Vasc Diffuse Lung Dis. 2009;26:155-161.

23. Kinder BW, Brown KK, McCormack FX, Ix JH, Kervitsky JA, Schwartz MI, et al. Serum surfactant protein-A is a strong predictor of early mortality in idiopathic pulmonary fibrosis. Chest. 2009;135:1557-63.

24. Satoh H, Kurishima K, Ishikawa H, Ohtsuka M. increased levels of KL-6 and subsequent mortality in patients with interstitial lung diseases. J Intern Med. 2006;260:429-34.

25. Prasse A, Probst C, Bargagli E, Zissel G, Toews GB, Flaherty KR, et al. Serum CC-chemokine ligand 18 concentrations predicts outcome in idiopathic pulmonary fibrosis. Am J Respir Crit Care Med. 2009;179:717-23. 